\title{
Recent Progress in Cost-effective and Stable AuAg/Cu-nanostructured Catalyst for Electrochemical Water Splitting
}

\author{
Received 23 November, 2020; revised 18 December, 2020; accepted 23 December, 2020
}

\author{
Abdul Qayoom Mugheria,** and Aijaz Ali Otho ${ }^{b}$ \\ a Dr. M.A Kazi Institute of Chemistry, University of Sindh, Sindh 76080, Pakistan \\ bInstitute of Plant Sciences, University of Sindh, Sindh 76080, Pakistan
}

*Corresponding author E-mail: a.qmugheri@scholars.usindh.edu.pk

\begin{abstract}
The design of an efficient cost-effective electrocatalyst that facilitates oxygen evolution reactions (OERs) in alkaline media is vital for present and future generations, with the durability and stability of the catalyst being of paramount importance. Such electrocatalysts are in high demand for renewable technologies, such as solar fuel development and fuel cells, as well as energy storage devices and lithium ion batteries. Herein, we present a simple wet chemical method for synthesizing trimetallic $(\mathrm{AuAg} / \mathrm{Cu})$ nanoparticles that can be utilized as an efficient electrocatalyst for OER. The prepared nanocomposite material delivers effective water splitting at a low overpotentials. The OER kinetics of the trimetallic composite material were analyzed experimentally in $1.0 \mathrm{M} \mathrm{KOH}$ electrolyte, revealing a Tafel slope of $82 \mathrm{mV} /$ dec corresponding to an overpotential of $300 \mathrm{mV}$ and a resistance of $177.9 \Omega$. These values provides the functional material for OER activity and can be used for electrochemical water splitting properties due to synergetic effects produced for the development of other functional materials composite material exhibiting excellent stability and durability over the 40 hours test duration. Therefore, this study demonstrates a safe and clean route toward fabricating efficient electrocatalysts comprising mixed-metal alloys that are suitable for renewable energy applications. The proposed synthesis method protocol is cost-effective and simple, and opens a new pathway for the preparation of efficient electrocatalysts.
\end{abstract}

Keywords: AuAg/Cu, Trimetallic alloy, Hydrogen evolution reaction, Kinetics, Oxygen evolution reactions

\section{Introduction}

Exacerbated by climate change and other environmental issues, the global energy crisis is one of the greatest challenges facing humanity. One promising and eco-friendly strategy to help resolve this challenge is the development of renewable hydrogen energy technology [1-3]. As such, many scientists have directed their attention to the production of hydrogen and oxygen. In industry, hydrogen is produced mostly via steam reforming of methane. However, hydrogen produced via this process has low purity owing to the presence of carbon residues; therefore, efficient water electrocatalysis has the potential to become a key technology in the future $[4,5]$. Water splitting is an effective method of generating pure hydrogen, where water is split electrochemically into hydrogen and oxygen $[2,3,6]$. Instigating an oxygen evolution reaction (OER) requires a large overpotential, which, in turn, requires a catalyst that initiates proton reduction and lowers the activation energy or overpotential $[7,8]$. The frequent use of electrocatalysts necessitates electrode materials with a large active surface area and high catalytic activity [9]. In addition, the catalyst must exhibit an elevated exchange current density and a small Tafel slope [10]. Metal-based compounds, such as $\mathrm{RuO}_{2}$ and $\mathrm{IrO}_{2}$, are considered the most effective catalysts and, therefore, widely used in OERs [2]. Moreover, oble metal catalysts like $\mathrm{Ru} / \mathrm{Ir}$ based materials are the state-of-art of catalysts for OER but they are very much costly and rare in nature andhave received close attention owing to their significant catalytic activity in basic media; unfortunately, their high cost and natural paucity have limited their appli- cation $[2,6,11]$. Consequently, the development of affordable alternatives to $\mathrm{RuO}_{2}$ and $\mathrm{IrO}_{2}$ is vital [11]. Alternative catalysts involving first row transition metal oxides perform poorly, highlighting the need for further studies of the OER kinetics of transition metal oxidebased materials at the nanoscale because, as particles decrease in size, their surface to volume ratio and catalytic properties increase [12]. By using nanotechnology, we can develop cheap electrocatalysts that enable the commercial production of oxygen from any source of water [13]. Although nanomaterial-based catalysts boost the efficiency of OERs, the scalability of the materials involved is also an important factor [14]. Previous studies have investigated electrocatalysts produced from earth-abundant elements including $\mathrm{Fe}, \mathrm{Ni}, \mathrm{Co}, \mathrm{Mo}$, and $\mathrm{W}$, as well as compounds such as molybdenum sulfides and carbides, and tungsten disulfide. Oxides comprising these elements offer several advantages; they are cheap, highly active, and exhibit a synergetic effect and fast charge transport toward OER kinetics in alkaline electrolytes $[7,8,15-17]$. Metal oxides, such as $\mathrm{NiO}_{\mathrm{x}}, \mathrm{MnO}_{\mathrm{x}}$, and transition metal-doped $\mathrm{TiO}_{2}$, have also been used as electrocatalysts for hydrogen and oxygen evolution reactions because of their electronic conductivity [18]. Furthermore, metal nanoparticles have been explored owing to their excellent physicochemical properties, such as quantum confinement, strong surface effects, and charge transport at the electrolyte-electrode interface, which indicate their suitability for catalysis [19]. Specifically, multi-metallic nanoparticles (TMNPs) have received great interest as a result of their unique catalytic, electrical, and optical properties among composite materials. In addition, the synergistic effects between nanoparticles comprising multiple 
metals are different from monometallic nanoparticles and make them excellent alternative electrocatalysts for water splitting [20]. In previous studies, different methods have been proposed for synthesizing TMNP-based nanocomposite materials, including microwave (MW) irradiation [21] and microemulsion systems [22]. In general, reducing the overpotential for OERs is a multi-stage process [23]. Nevertheless, the synthesis of $\mathrm{AuAg} / \mathrm{Cu}$ nanoparticles for use in water splitting is yet to be reported. This is a simple approach, which can be applied at low temperatures via the aqueous chemical growth method. To enhance OER activity kinetics, we used temperature annealing in air, which has never been reported previously for $\mathrm{AuAg} / \mathrm{Cu}$ nanoparticles. The fabricated $\mathrm{AuAg} / \mathrm{Cu}$ particles possess increased conductivity and surface area. Furthermore, they deliver improved catalytic performance, thereby facilitating increased OER activity. Further advantages include excellent stability and the lowest Tafel slope value reported for an electrocatalyst in alkaline media to date.

Here, we report the fabrication of hetero-structured materials via a simple wet chemical method and an easy stepwise procedure for the synthesis of trimetallic alloy composites. The synthesized trimetallic nanoparticles show impressive catalytic activity with respect to OERs in basic media, demonstrating that this new pathway for fabricating electro-catalysts may be applied across diverse energy sectors.

\section{Experimental details}

\subsection{Materials}

Hydrogen tetrachloroauratetrihydrate $\left(\mathrm{HAuCl}_{4} \cdot 3 \mathrm{H}_{2} \mathrm{O}\right)$, silver nitrate $\left(\mathrm{AgNO}_{3}\right)$, copper acetate monohydrate $\mathrm{Cu}\left(\mathrm{CH}_{3} \mathrm{COO}\right)_{2}$, and hex amethyl tetra amine (HMT) $\left(\mathrm{C}_{6} \mathrm{H}_{12} \mathrm{~N}_{4}\right)$ were used directly without further purification. All glassware were cleaned with aqua regia and then rinsed with distilled water.

\subsection{Synthesis of Au, Ag, and Cu nanostructured com- posite}

Step-1: $\mathrm{CuO}$ nanostructures were grown at a very low temperature via an aqueous chemical growth method. First, $0.1 \mathrm{M}$ of copper acetate monohydrate, $1.6 \mathrm{~g}$ of hexamethylenetetramine, and $10 \mathrm{~mL}$ of ammonia were dissolved in $90 \mathrm{~mL}$ of distilled water. The homogeneous solution was covered with aluminum foil at $90{ }^{\circ} \mathrm{C}$ in an electric oven for $5 \mathrm{~h}$. The resulting obtained material of copper hydroxide nanostructure was collected and left to air for $12 \mathrm{~h}$. for properly draying Then, the $\mathrm{CuO}$ nanostructure was functionalized with $\mathrm{Au}$ and $\mathrm{Ag}$ via the wet chemical method.

Step-2: $\mathrm{AuAg} / \mathrm{Cu}$ nanocomposites were prepared using the hydrothermal method. A solution comprising gold chloride $(0.34 \mathrm{~g})$, silver nitrate $(0.22 \mathrm{mg})$, HMT $(1.6 \mathrm{~g})$, and hydrochloric acid $(5 \mathrm{~mL})$ added to $70 \mathrm{ml}$ of deionized water was prepared and divided between two beakers, to which 0.1 (sample S1) and $0.2 \mathrm{~g}$ (sample S2) of $\mathrm{CuO}$ were added; both mixtures were stirred for $1 \mathrm{~h}$. The trimetric mixtures were transferred into a $100 \mathrm{~mL}$ Teflon-lined stainless-steel autoclave at a temperature of $250^{\circ} \mathrm{C}$ for $12 \mathrm{~h}$ and cooled at room temperature (the emergence of a light purple color indicates the formation of particles). The precipitates of the nanocomposite were collected and washed several times with deionized water and alcohol separetely. The nanocomposite material was dried at $70{ }^{\circ} \mathrm{C}$ overnight. The Pristine (AuAg) composite material was prepared via the same procedure without the addition of the $\mathrm{CuO}$ material.

\subsection{Modification of glassy carbon electrode with syn- thesized AuAg/Cu trimetallic composite alloy}

To optimize the trimetallic system for water splitting and verify the stability of OERs in the nanocomposite system, the functional results of the electrocatalysts were confirmed. First, an electrode was cleaned with alumina powder before being washed with ethanol and water by sonication. Then, the electrode was dried and modified. To modify the glassy carbon electrode, $15 \mathrm{~mL}$ of the prepared nanoparticles was dropped on the glassy carbon electrode surface using the drop-casting method. After drying at room temperature, $5 \mathrm{~mL}$ of Nafion solution $(0.5 \%)$ was dropped onto the composite material as a binder. The oxygen evolution activity of the electrodes modified by the $\mathrm{AuAg} / \mathrm{Cu}$ nanoparticles composite alloy was measured using an electrochemical approach in which platinum wire was used as the auxiliary electrode and a calomel electrode was used as a reference electrode in alkaline media. Electrochemical experiments were conducted over a frequency range spanning $100 \mathrm{kHz}$, with an amplitude of $10 \mathrm{mV}$ at a applied potential of $1.53 \mathrm{~V}$. The nanostructure composite material exhibiting the best OER kinetic activity was identified and all the experiments were repeated 6 times. Consequently, electrochemical impedance spectroscopy (EIS) results obtained using Z-view software and the fitted electrolysis results at a static overpotential of 1.23 $\mathrm{V}$ are presented, confirming the material results.

\subsection{Experimental conditions for structural charac- terization}

A transmission electron microscope (TEM) equipped with a field emission gun was used to acquire TEM images. It was operated at 200 $\mathrm{kV}$ with a point resolution of $1.9 \AA$ and used in conjunction with an energy-dispersive spectrometer (EDS). The nanocrystalline structure of the samples was investigated using X-ray diffraction (XRD; Philips PW 1729), while Fourier transform infrared spectroscopy (FTIR) was used to identify the interaction between aspirin and the trimetallic nanoparticles. UV-visible spectra (Cary 100 UV-Vis) were also recorded.

\section{Results and discussion}

\subsection{Characterization of trimetallic AuAg/Cu nanopar- ticles}

Figure 1(a) and 1(b) show the EDS-TEM and TEM images, respectively, of the trimetallic composite, with the EDS result showing the distributions of $\mathrm{Au}, \mathrm{Ag}$, and $\mathrm{Cu}$. The TEM images indicate that the metallic composite is involved in the alloy formation, as evidenced by Fig. 1(b). The TEM images confirm that the average size of the trimetallic nanoparticles ranges from $30-50 \mathrm{~nm}$.

The crystalline nature of the nanoparticles was investigated using XRD, with Fig. 1(c) showing the XRD pattern for the aspirin-capped trimetallic nanoparticles. The XRD pattern in Fig. 1(c) shows peaks at $36.40^{\circ}, 42.44^{\circ}, 44.26^{\circ}$, and $63.5^{\circ}$, which represent the (111), (200), (220), and (311) crystal planes of the face-centered cubic structure of the nanocomposite material; these peaks are compatible with the AuAg component of the structure. Further peaks represent the reflections from the (111), (200), and (220) planes in $\mathrm{Cu}$. All peak positions are intermediate to those of $\mathrm{AuAg}$ and $\mathrm{Cu}$. The diffraction patterns obtained in the XRD experiment provide conclusive evidence for the formation of $\mathrm{AuAg} / \mathrm{Cu}$ alloys. Additionally, the XRD reveals that the prepared trimetallic nanoparticles samples do not contain any $\mathrm{Cu}_{2} \mathrm{O}$ or $\mathrm{CuO}$ impurities. The XRD results agree fully with measurements made from the TEM images. Next, Fig. 1(d) shows the surface plasmon resonance band of spherical the $\mathrm{AuAg} / \mathrm{Cu}$ nanoparticles. The peak indicating maximum absorption is located at $571 \mathrm{~nm}$, as shown in Fig. 1(d). Figure 2 presents an atomic force microscopy (AFM) image that describes the surface morphology of the trimetallic nanoparticles. The AFM analysis reveals that trimetallic nanoparticles are spherical and distributed uniformly. Furthermore, the presence of cluster or aggregate formation can be observed. The trimetallic nanoparticles have an average diameter ranging from $26-35 \mathrm{~nm}$, showing further agreement with TEM observations. In addition, the UV spectra show the 

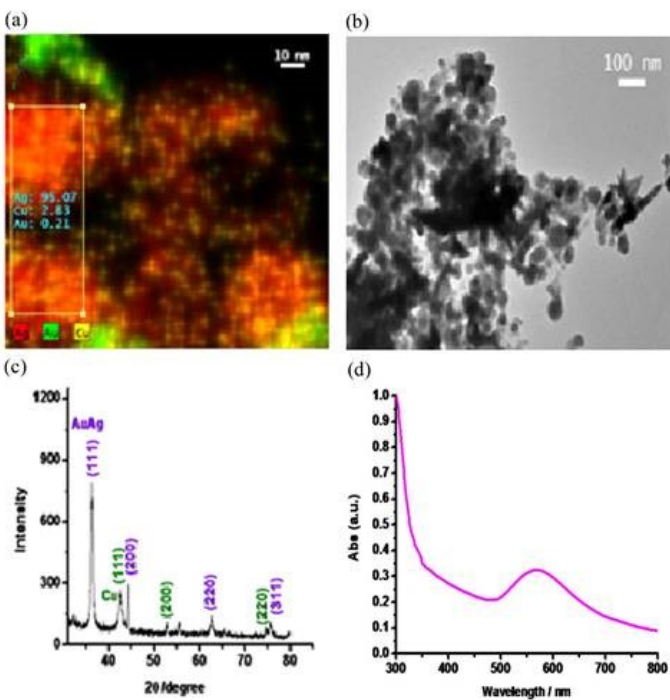

Figure 1. (a,b) TEM images of trimetallic nanoparticles. (c) XRD pattern. (d) UVvisible spectrum.

optical characteristics of each metal component in the nanocomposite material. Peaks are observed at 560, 400, and $600 \mathrm{~nm}$, corresponding to $\mathrm{Au}, \mathrm{Ag}$, and $\mathrm{Cu}$, respectively [24-26].

As shown in Fig. 3, the elemental analysis performed via EDS confirms the presence of the three metals, i.e., $\mathrm{Au}, \mathrm{Ag}$, and $\mathrm{Cu}$, in the nanostructured composite material. The EDS also provides the percentage of each element in the composite material, which is $56.45 \%$ $\mathrm{Au}, 23.79 \% \mathrm{Ag}$, and $19.76 \% \mathrm{Cu}$.

\subsection{Electrocatalyst performance of AuAg/Cu nano- al-loy for OER activity}

The electrochemical water splitting proved that the composite material have low charge transfer resistance, which confirms the excellent connection between the composite electrodes and electrolyte. These composite electrodes exhibit externally and further increase the excellent outperforming for OER due to surface active sites, which enhance the Tafel slope comparable, or even lower than commercial Ru-based electrodes, which is unprecedented for non-noble electrocatalysts at the acceptable onset potential.

The nanocomposite is used to modify the glassy surface of the carbon electrode, thereby providing the electrode with greater contact area between the electrocatalyst materials that enhance the transport and capture of target molecules in the area of the electrode and promote the electron transfer rate. To modify the glass carbon electrode, 5 $\mathrm{mg}$ of the composite material was dispersed in $3 \mathrm{~mL}$ of deionized water and $15 \mu \mathrm{L}$ of freshly prepared catalyst ink. The polarization curves for pristine AuAg and the electrode modified with trimetallic nanoparticles are shown in Fig. 4. The $\mathrm{AuAg} / \mathrm{Cu}$ catalyst shows nearly negligible overpotential and potential OER activity for the trimetallic nanoparticles compared to the mono and bimetallic nanoparticles. The polarization curves show the excellent oxygen evolution properties of the composite catalysts (Fig. 4), with the OER mechanism involving three steps: (i) adsorption (Volmer step), (ii) desorption (Heyrovsky step), and (iii) Tafel slope. Each of these steps helps to determine the general rate of reaction (1). In step (i), hydrogen ions are adsorbed to the composite surface, while in step (ii), hydrogen ions gain electrons, produce hydrogen gas, and are discharged from the surface of the modified electrode. The specifics of the water splitting process using the newly synthesized composite electrocatalyst are shown by the following reactions:

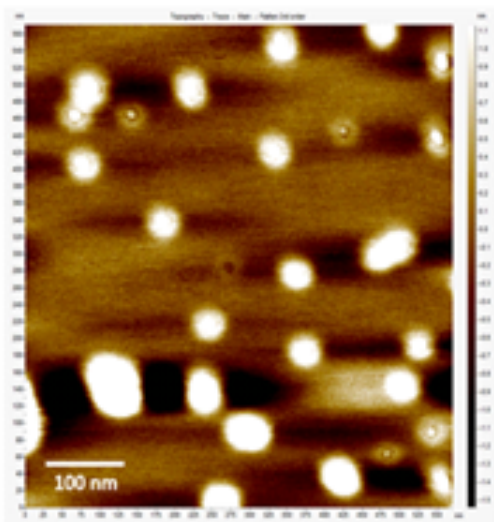

Figure 2. AFM image of the trimetallic AuAg/Cu nanoparticles.

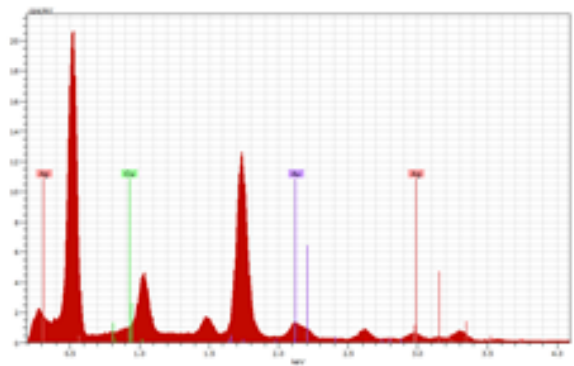

Figure 3. EDS spectrum of the trimetallic nanocomposite material.

$$
\begin{aligned}
2 \mathrm{H}_{2} \mathrm{O}+2 e^{-}+2 \mathrm{M} & \rightarrow 2 \mathrm{MH}_{(\text {ads })}+2 \mathrm{OH}, \\
2 \mathrm{MH}_{(\text {ads })}+2 \mathrm{H}_{2} \mathrm{O}+2 e^{-} & \rightarrow 2 \mathrm{H}_{2}+2 \mathrm{OH}+2 \mathrm{M}, \\
2 \mathrm{MH} & \leftrightarrow \mathrm{H}_{2}+2 \mathrm{M} .
\end{aligned}
$$

Through alkaline water electrolysis, pure hydrogen gas can be produced without emitting carbon residues. For the hydrogen evolution reaction (HER), the Tafel slope is an important step, through which the reaction mechanism of the catalyst is determined. If the Tafel slope value is $82 \mathrm{mV} /$ decade, then desorption (Volmer-Heyrovsky) is the rate-determining step, otherwise the Tafel recombination step (Volmer-Tafel) is the rate-determining step. Tafel slopes serve a practical purpose as the prepared $\mathrm{AuAg} / \mathrm{Cu}$ electrocatalyst exhibits good catalytic activity and shows a comparable Tafel slope, as shown in Fig. 4(b). Upon inspection of the OER activity of the glassy carbon electrode for the electrocatalyst, we observe the generation of oxygen gas at more positive overpotentials compared to the $\mathrm{AuAg} / \mathrm{Cu}$ trimetallic nanoparticle alloys. Thus, these results highlight the synergic role of $\mathrm{Ag}$, while the addition of $\mathrm{Au}$ to $\mathrm{Cu}$ lowers the overpotential barrier owing to its outburst catalytic property. However, $\mathrm{Cu}$ enhances the electrical conductivity and improves the surface area for the materials, resulting in unique OER activity that demonstrates the promise of this electrocatalyst. To explore the outstanding properties of this material in greater depth, it is important to study the elementary reactions steps in the OER process. The OER kinetics are governed by the Tafel slope. The OER kinetics of the prepared trimetallic alloy outperform electrocatalysts reported previously. The simple splitting of the reactants to produce reaction intermediates and the spontaneous collection of these intermediates are crucial parameters in achieving an energetic and specific catalysis process. The addition of $\mathrm{CuO}$ to $\mathrm{Ag}$ and $\mathrm{Au}$ in the formation of the $\mathrm{AuAg} / \mathrm{Cu}$ alloy does not require optimization in order to expose the features of $\mathrm{Ag}$ and $\mathrm{Cu}$ simultaneously. 


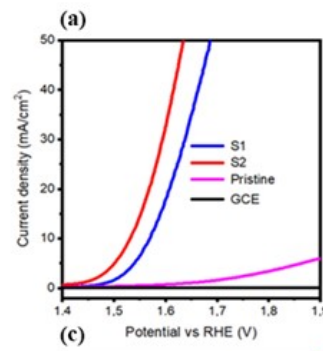

(b)
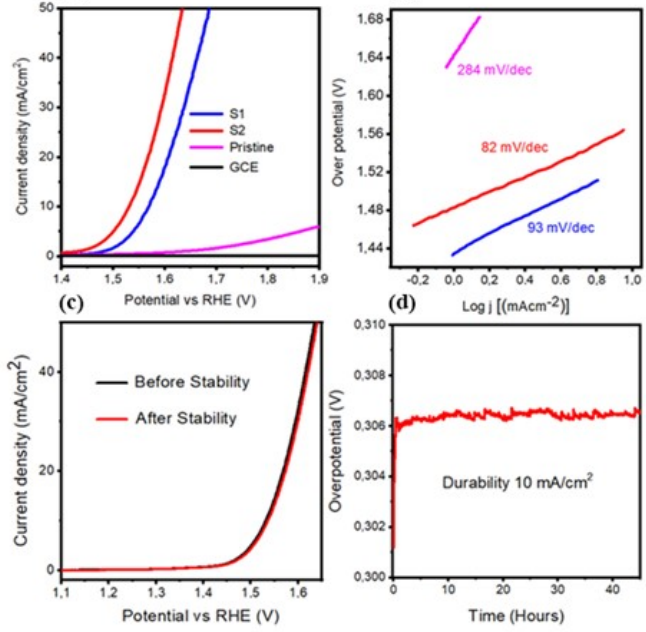

(d) $\quad \log \mathrm{i}\left[\left(\mathrm{mAcm}^{-2}\right)\right]$

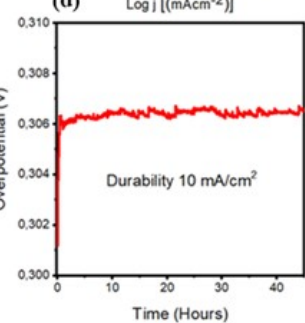

Figure 4. (a) LSV polarization curves of pristine cobalt oxide, S1 and S2 at scan rate of $1 \mathrm{mV} / \mathrm{s}$ in $1 \mathrm{M} \mathrm{KOH}$, (b) Tafel plots from linear region of polarization curves, (c) LSV polarization curves of stability for S2, and (d) chronopotentiometry experiment for $40 \mathrm{~h}$ durability response at $10 \mathrm{mAcm}^{2}$.

Therefore, the efficiency of the OER for the trimetallic AuAg/Cu electrocatalyst is most likely associated with multifunctional aspects. For example, the edges of Ag permit the splitting of water and the generation of hydrogen and oxygen intermediates, which can be adsorbed by the surface of $\mathrm{Au}$ and recombined to form diatomic hydrogen or oxygen [6]. In addition, $\mathrm{Cu}$ atoms tend to reduce the energy barrier required to produce hydrogen intermediates and, subsequently, facilitate desorption from the surface of $\mathrm{Cu}$ atoms, while accelerating the electron transfer rate [7] and enhancing the oxygen evolution performance. The improved HER kinetics can be attributed to the electronic structure of the AuAg-rich surface in the trimetallic $\mathrm{AuAg} / \mathrm{Cu}$ alloy that not only hinders the possibility of transition metal solubility but also generates additional surface activity. The improved electron coupling interaction between $\mathrm{Au}, \mathrm{Ag}$, and $\mathrm{Cu}$ endorses the lower exposure of reduced intermediates due to poor oxygen-binding strength, which arises following the decrease of $4 \mathrm{f}$ binding energies in Au. In summary, we conclude that the inner contact between the $\mathrm{Au}, \mathrm{Ag}$, and $\mathrm{Cu}$ areas in the trimetallic alloy significantly accelerates the reclamation of $\mathrm{Au}$ sites that contribute to the electrochemical activity, which enhances the OER performance [8-17]. The performance of these nonprecious trimetallic alloys is excellent relative to the alternative materials that have been reported in literature. Stability and durability are key factors in determining the practical applications of electrocatalysts. In addition to having excellent OER activity, the trimetallic $\mathrm{AuAg} / \mathrm{Cu}$ modified electrode also exhibits high durability, showing little sign of degradation over 1000 cycles in the selected range over a period of $40 \mathrm{~h}$ [see Fig. 4(d)] durability response at $10 \mathrm{mAcm}^{2}$ as shown in Fig. 4(d). Composite nanostructures are used as potential candidates in diverse applications, is excellent and it revealed the potential capability of $\mathrm{S} 2$ to retain the OER activity for long period. The low Tafel value for noble metal free catalyst in OER is clear evidence for its promising practical applications in alkaline media and can be capitalized for industrial purposes. Also, this study provides a solid platform for the strengthening of oxygen economy using metals alloys as a gate way. To demonstrate the robust overall water splitting composite is strongly regarding OER activities using electrochemical approach lowers the barrier of high overpotentials by the mixed metal oxide and composite phase-in achieving the low Tafel slope values and huge current density in basic media. Thus demonstrating the long-
Table 1. Ans: The fitted values for equivalent circuit of elements.

\begin{tabular}{cccc}
\hline & $\begin{array}{c}\text { Static } \\
\text { resistance } \\
(\Omega)\end{array}$ & $\begin{array}{c}\text { Charge } \\
\text { transfer } \\
\text { resistance } \\
(\Omega)\end{array}$ & $\begin{array}{c}\text { Double- } \\
\text { layer } \\
\text { capaci- } \\
\text { tance }(\mathrm{F})\end{array}$ \\
\hline Pristine Composite & 8.65 & 1879 & 168.51 \\
Sample S1 & 8.61 & 339.8 & 122.20 \\
Sample S2 & 8.58 & 177.9 & 232.66 \\
\hline
\end{tabular}

term stability and scalability of the newly developed OER catalyst. The durability of the $\mathrm{AuAg} / \mathrm{Cu}$ composite was tested via chronopotentiometry using current densities of 10 and $20 \mathrm{~mA} / \mathrm{cm}^{2}$. Moreover, stability and durability studies were also conducted for sample S2 to ensure its application potential. The Tafel slopes and polarization curves support the kinetics of the OER at the electrode-electrolyte interface. Electrochemical impedance spectroscopy was also performed for the frequency range from $100 \mathrm{kHz}$ to $0.1 \mathrm{~Hz}$ at the amplitude of $10 \mathrm{mV}$ and an onset potential of $1.53 \mathrm{~V}$ vs reversible oxygen electrodes (RHE) in 1.0 M KOH electrolyte. At the high-frequency semicircle, an arc is indexed to the charge transfer resistance, which is related to the exchange current density. Electrochemical impedance spectroscopy was utilized to evaluate the kinetics of the OER in catalysts, as shown in Fig. 4(c). The elements were modelled by two RC components in the unified equivalent circuit, which was developed for an electrode protected by a damaged coating $[18,19,25]$, while we also utilized the fitting values of the catalyst spectra of the modified electrode. The lack of layer smoothness in the proper pattern is attributed to the improved surface roughness with anodization time; analyzing the modified electrode via the impedance response is complex, with the element phase circulated with a circuit model featuring constant phase elements rather than pure capacitors. The constant phase element (equivalent circuit) providing the finest fit consists of the electrolytic solution resistance $R_{s}$ and two joint R-CPE units (I and II). The charge transfer resistance for pristine AuAg is $1879 \Omega$, with samples S1 and S2 having charge transfer resistances of 339.8 and $177.9 \Omega$, respectively. The fitted values for the composite nanostructure and the pristine $\mathrm{AuAg}$ are shown in Table 1 alongside their equivalent circuit values. The impedance frequency values range from $100 \mathrm{kHz}$ to $0.1 \mathrm{~Hz}$. The real and imaginary values of the spectra indicate a significantly better fit. The charge transfer resistance estimated for sample S2 $(177.9 \Omega)$ was less than that for sample $S 1(339.8 \Omega)$, while the rest of the fitting parameters revealed only minor changes. This illustrates that the OER occurs faster on the nanostructured sample S2, with the higher capacitance, than on sample S1, indicating the improved capability of sample S2 to transfer ionic charge between the electrolyte and electrode, as shown in Fig. 4(a). The surface area of the nanocomposite material was assessed by cyclic voltammetry against $\mathrm{Ag} / \mathrm{AgCl}$ by considering a non-faradaic part, as shown Fig. 5. This result can be attributed to the porous structure of cobalt oxide nanowires, which result from the self-assembly of nanoparticles of cobalt oxide, as can be seen from low-resolution TEM studies. The OER polarization curves and Tafel slopes at the electrode-electrolyte interface were studied in greater detail via an electrochemical impedance experiment. Owing to the configuration of this catalyst, the low frequency linear region is devoted to the high-frequency semicircle arc, which is indexed for the charge transfer resistance. A significant change in the OER activity was observed, which indicates good chemical and structural stability. As mentioned previously, these are attractive and unique functional properties, which significantly enhance water splitting in alkaline media. The cyclic voltammetry analysis revealed the active surface area properties of the nanocomposite material at different scan rates, thereby confirming material stability. The chronopotentiometry test demonstrated the durability of the nanocomposite over a 40 $\mathrm{h}$ period. EIS was used to study the electrochemical impedance of the composite material, revealing a small charge transfer resistance of 

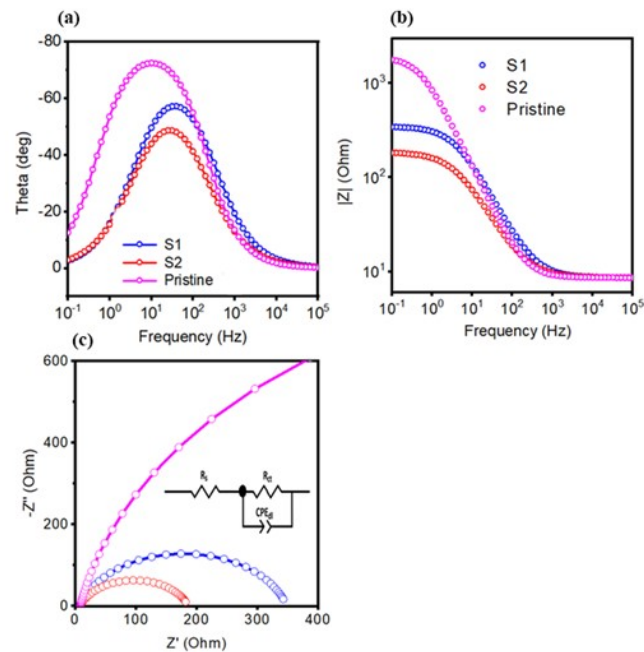

Figure 5. Electrochemical impedance spectroscopy experiment for the frequency range $100 \mathrm{kHz}$ to $0.1 \mathrm{~Hz}$, at amplitude of $10 \mathrm{mV}$ and onset potential of $1.53 \mathrm{~V}$ vs RHE in $1 \mathrm{M} \mathrm{KOH},(\mathrm{a}, \mathrm{b})$ Bode plots and (c) Nyquist plots

$177.9 \Omega$ and a higher capacitance value. We demonstrated the fabrication of a trimetallic $(\mathrm{AuAg} / \mathrm{Cu})$ nanocomposite material, which represents a promising alternative eco-friendly OER catalyst for water oxidation and other numerous energy conversion and storage applications. This is the first report that presents a low-cost electrocatalyst for rapid OERs. Therefore, the proposed trimetallic alloy should be of great interest as a catalyst in the fields of energy harvesting and renewable energy. In addition, the impressive performance of this nonprecious trimetallic alloy opens a new avenue in the engineering of cost-effective electrocatalysts and provides a solid platform for the strengthening of hydrogen-based technologies. To date, no attention has been paid to the excellent features achievable by coupling trimetallic oxides with stannous compounds using an Au element to achieve an active OER.

\section{Conclusions}

In summary, we synthesized an $\mathrm{AuAg} / \mathrm{Cu}$ alloy via a wet chemical method to produce an electrocatalyst for OER in alkaline media. The resulting trimetallic nonprecious alloy catalyst delivers high OER activity with negligible overpotential and a small Tafel slope. Experiments were performed in alkaline media at a current density of 10 $\mathrm{mA} / \mathrm{cm}^{2}$ and a potential of less than $300 \mathrm{mV}$. These findings provide a simple and cost-effective electrocatalyst that delivers a superb OER performance. These impressive properties are attributed to the $\mathrm{AuAg} / \mathrm{Cu}$ mixed-metal alloy in which the interplay between the different elemental constituents creates a synergic effect. The low overpotential, small Tafel slope, and low charge transfer resistance are the attractive features of the sample $\mathrm{S} 2$ as compare to precious metal $(\mathrm{RuO})$ catalyst in the OER is clear evidence for its applicability in alkaline media, which is immensely promising for industrial purposes. As such, this study provides a platform for the development of inexpensive, environmentally benign, and efficient electrocatalysts that can strengthen the oxygen economy, with the $\mathrm{AuAg} / \mathrm{Cu}$ nanocomposite serving as a gateway for the development of the next generation of electrocatalysts and energy conversion technologies.

\section{Acknowledgements}

I look forward to justice for chairman cadet colleges 16 division pano akil and efforts will be generated for this justice.

\section{References}

[1] M. G. Walter, E. L. Warren, J. R. Mc-Kone, S.W. Boettcher, Q. Mi, E. A. Santori, and N. S. Lewis, Chem. Rev. 110, 6446 (2010).

[2] Y. Li and C. Zhao, Chem. Mater. 28, 5659 (2016).

[3] J. Wang, W. Cui, Q. Liu, Z. Xing, A. M. Asari, and X. Sun, Adv. Mater. 28, 215 (2016).

[4] S. Pawar et al., J. Mater. Chem. A 5, 12747 (2017).

[5] N. S. Lewis and D.G. Nocera, Proc. Natl. Acad. Sci. U.S.A 113, 15729 (2006).

[6] F. Cheng and J. Chen, Chem. Soc. Rev. 41, 2172 (2012).

[7] H. B. Gray, Nat. Chem. 1, 7 (2009).

[8] A. Kudo and Y. Miseki, Chem. Soc. Rev. 38, 253 (2009).

[9] Y. Liang, Y. Li, H. Wang, and H. Dai, J. Am. Chem. Soc. 135, 2013 (2013).

[10] Y. C. Lu, Z. Xu, H. A. Gasteiger, S. Chen, K. Hamad-Schifferli, and Y. Shao-Horn, J. Am. Chem. Soc. 132, 12170 (2010).

[11] R. D. Smith, M. S. Prevot, R. D. Fagan, Z. Zhang, P. A. Sedach, M. K. Siu, S. Trudel, and C. P. Berlinguette, Science 340, 60 (2013).

[12] H. Wang and H. Dai, Chem. Soc. Rev. 42, 3088 (2013).

[13] S. W. Lee, C. Carlton, M. Risch, Y. Surendranath, S. Chen, S. Furutsuki, A. Yamada, D. G. Nocera, and Y. Shao-Horn, J. Am. Chem. Soc. 134, 16959 (2012).

[14] T. A. Betley, Q. Wu, T. V. Voorhis, and D. G. Nocera, Inorg. Chem. 47, 1849 (2008).

[15] Q. Lu et al., Nat. Commun. 6, 6567 (2015).

[16] F. Safizadeh, E. Ghali, and G. Houlachi, Int. J. Hydrog. Energy 40, 256 (2015).

[17] X. M. Goh, Y. Zheng, S. J. Tan, L. Zhang, K. Kumar, C. W. Qiu, and J. K. W. Yang, Nat. Commun. 5, 5361 (2014).

[18] V. Viswanathan, K. L. Pickrahn, A. C. Luntz, S. F. Bent, and J. K. Nørskov, Nano Lett. 14, 5853 (2014).

[19] S. Sareen, V. Mutrejab, S. Singha, and B. Pal, RSC Adv. 5184 (2015).

[20] P. Qiao, S. Xu, D. Zhang, R. Li, S. Zou, J. Liu, W. Yi, J. Li, and J. Fan, Chem. Commun. 50, 11713 (2014).

[21] B. Loganathan and B. Karthikeyan, Colloids Surf. A Physicochem. Eng. Asp. 436, 944 (2013).

[22] X. Zhang, F. Zhang, and K. Y. Chan, Catal. Commun. 5, 749 (2004).

[23] B. Zhao et al., Nat. Commun. 8, 14586 (2017).

[24] T. Shinagawa, A. T. Garcia-Esparza, and K. Takanabe, Sci. Rep. 5, 13801 (2015)

[25] S. T. Christensen, H. Feng, J. L. Libera, N. Guo, J. T. Miller, P. C. Stair, and J. W. Elam, Nano Lett. 10, 3047 (2010).

[26] N. Cheng, Y. Xue, Q. Liu, J. Tian, L. Zhang, A. M. Asiri, and X. Sun, Electrochim. Acta 163, 102 (2015).

[27] X. Liu, S. Cui, Z. Sun, Y. Ren, X. Zhang, and P. Du, J. Phys. Chem. C. 120, 831 (2016)

[28] X. Liu, S. Cui, Z. Sun, and P. Du, Electrochim. Acta. 160, 202 (2015).

[29] A. D. Handokoet al., Catal. Sci. Technol. 6, 269 (2016).

[30] K. S. Joya and H. J. M. de Groot, ACS Catal. 6, 1768 (2016).

[31] F. Song and X. Hu, Nat. Commun. 5, 4477 (2014).

[32] H. Jin, J. Wang, D. Su, Z. Wei, Z. Pang, and Y. Wang, J. Am. Chem. Soc. 137,2688 (2015).

[33] F. Song and X. Hu, J. Am. Chem. Soc. 136, 16481 (2014).

[34] Y. Liang, Y. Li, H. Wang, J. Zhou, J. Wang, T. Regier, and H. Dai, Nat. Mater. 10, 780 (2011)

[35] C. L. Charles, S. McCrory, C. J. Jung, F. Peters, and T. Jaramillo, J. Am. Chem. Soc. 135, 16977 (2013). 